\title{
Research on the Application Prospect of Big Data Technology in Intelligent Transportation
}

\author{
Yan Hou \\ Qilu Normal University, Jinan, Shandong, 250014, China
}

\begin{abstract}
In the new era of Internet and big data, urban traffic has been effectively relieved, which mainly benefits from the reasonable application of big data technology. The fully expression of application advantages of big data has improved urban traffic jam and helped intelligent transportation service more perfect, guaranteeing the intelligent development of urban traffic transportation. This paper will mainly analyze the application of big data technology in intelligent transportation and understand the current status of heavy urban traffic combining with present urbanization. And the scientific plan of its application prospect will be made to timely relieve such situation and improve the service model of intelligent transportation.
\end{abstract}

Keywords: big data; intelligent transportation; application prospect.

\section{Introduction}

At present, the intelligent transportation system tends to be improved, and the collection means used by video images are widely used in various fields, including electronic police, illegal monitoring and so on. The emergence of this application trend reflects the importance of big data technology in intelligent transportation. Even though the present effect is remarkable, there still exist many places to be improved. And the steady improvement of acquisition quality should be focused on so as to guarantee the rational use of big data technology and promote the steady enhancement of service level of intelligent transportation.

\section{Basic Overview of Big Data Technology}

The big data technology is to ensure efficient information filtering in lots of data and to provide reliable help for solving big data problems. The reasonable use effect of big data technology is remarkable. And there are two parts that should be rational mastered: the first one is making effective filtering with useful information from lots of data information; and the second one is to gradually broaden the basics methods of big data research and development, mainly including the establishment of analysis data model, storage and data processing.

\section{The Application Need of Big Data Technology in Intelligent Transportation}

\subsection{Focus on the Promotion of Timeliness and Initiative}

Lots of people crowd into the city in the development process of urbanization, and most cities face immense traffic pressure, which heavily affect the total process of city development. At present, the big data referred in the transportation shows the basic characteristics such as high-speed and diversified. Its pattern and data distributed degree set the hindrance for the storage and processing. It needs to take rather long time, which totally disobeys the basic demand of traffic timeliness. Intelligent transportation also advocate the change with former passive model about the processing of related data information.

\subsection{Focus on the Promotion of the Ordering and Difference}

Faced with the coming of big data era, the development of e-commerce has developed rapidly, which meets the demand of people's online shopping and pushes the progress and development of logistics industry to some degree. In the present era paying attention to the urban traffic, the smoothness of delivery process will directly relate to the healthy development of urban economy. 
Viewed from now, national logistics delivery development still cannot cater to the higher demand with delivery, which will threaten urban economy moving for better level. The reason is the irrational routines of urban delivery plan so as to affect the delivery efficiency. Besides, lacking enough attention to the differences of users has resulted in that the delivery service cannot timely arrive and the low peak and high peak have remarkable difference in the logistics delivery.

\section{Significant Influence of Big Data Technology Application to the Intelligent Transportation}

\subsection{Virtuality of Big Data Technology}

In the new era, the birth and development of big data technology have injected vitality into the development of intelligent transportation, especially its own virtuality, which can timely cope with the existing crossing problems among administrative areas. Transportation big data should strictly obey the basic rule of information communication and sharing so as to take effective countermeasures to solve the information crossing-domain problems.

\subsection{Big Data can Complete the Integration and Combing of Information}

In the process of specifically implementing the intelligent transportation service, the advantage of big data technology should be resorted to timely deal with acquired information, accelerate the construction rate of transportation system and construct rather complete information integration model in the process of integrating many regional data base. The construction of such model can ensure the full manifestation of transportation functions and potential values to further create more reliable development opportunities. For instance, properly combining with specific data resources of weather and transportation can timely avoid various catastrophes that may exist in the specific regions and make the distributed conditions of urban transportation more clear through the integration with various information collected by the password card such as RFID.

\subsection{Intelligent Transpiration Requires Intelligence}

The further development of intelligent transportation needs to resort to the intelligence of big data to easily optimize and integrate various referred transportation resources and better allocate them. Dealing with different data through rational analysis will be good for the smooth launching of transportation management and implementation and effective coordination plan. The reasonable analysis with big data implementation can reduce the input of related departments in the practical operation to more scientifically use various transportation resources. For instance, the analysis with relevant results of big data should focus on the optimization allocation of bus network and make the passage control of bus network and traffic information after clarifying the scientific and effective flow organization scheme.

\subsection{Big Data can Realize Quick Forecast}

As for intelligent transportation service, the application of big data technology can meet with the quick forecast of related information and make forecast level get obvious enhancement through the application of this technology. Extract the related data involved in different departments and construct rather rational traffic forecast model which can correctly analyze practical operation condition of transportation and timely check the trip plan. Aiming at the forecast of various traffic field, big data can more scientifically judge practical road condition to analyze the probability of car collision and forecast the condition such as drivers' lane change, owing to the function of forecast and properly coping with information.

\subsection{Big Data Technology Can Improve the Operational Efficiency}

Proper application of big data technology in the intelligent transportation can timely improve operational efficiency and guarantee good passage ability of road net and cater to the adjustment 
demand of traffic efficiency at the same time of properly adding facilities. In order to improve traffic conditions, the great volume feature of big data can relieve the working difficult of related projects, which reduces the input of some capitals to some degree.

\section{Intelligent Transportation Put Forward New Requirements for Big Data Technology}

\subsection{Change Former Transportation Guidance Means}

Currently, the occupation rate of urban cars tends to be gradually saturated and even appears rather heavy traffic jam and threaten the normal travel of urban residents. Compared with data information of other types, transportation data shows the characteristics of great scale, distributed storage and various patterns. And the analysis period of data processing is rather long, so its application should focus on changing former transportation guidance methods and transform the negative and lagged guidance to positive and instant guidance.

\subsection{Urban Delivery Methods Should Tend to be Diversified}

Nowadays, Alibaba groups have experienced rapid development and e-commerce has appeared the booming development trend, so intelligent transportation claims to change urban delivery methods when applying the big data technology. Development degree of logistics enterprises is not corresponding with urban delivery standards, which hinders the steady development of urban economy from the side. Because of long time in filtering and delivery routines, current domestic urban delivery routine plan affects the transportation efficiency. So, it holds that the application of big data technology to the intelligent transportation should realize the diversified urban delivery to satisfy the high demand for urban development.

\section{The Application Prospect of Big Data Technology in Intelligent Transportation}

\subsection{Complete Institutionalized and Standardized Construction}

The government attaches great importance to the construction of intelligent transportation, so it has regulated relevant legal system and strengthened basic legislative work. Today, under the reasonable use of big data technology, in order to ensure the smooth development of the intelligent construction, it is advocated to strengthen construction of standardization and institutionalized through these regulations so as to make the corresponding more smooth.

\subsection{Implement the Training of Large Numbers of Talents}

In order to better realize the intelligent transportation and analyze the application value combined with large data technology, we need to cultivate more and more excellent talents to master solid theoretical knowledge and practical experience, so as to better devote themselves to the follow-up work. People in the age of big data should have comprehensive knowledge of information, transportation and management. In order to ensure the great value of large data technology in the intelligent transportation, it is advocated to enhance the personnel quality, strengthen the strategy of personnel training, and select high-quality personnel, and thus to consolidate the application effect of the large data.

\subsection{Optimize Real-Time Traffic Services}

When optimizing the traffic problems involved, we should pay more attention to the advantages of the application of large data technology, such as the proper breakthrough of the limitations of across the region and the steady improvement of the efficiency of information integration, as well as the scientific allocation of related resources. The large data technology itself shows the real time, and when monitoring the actual traffic situation in the city, we could plan the most reasonable route 
properly so that the ideal effect can be achieved. The analysis of the traffic problems by big data technology can help to dredge the road, relieve the traffic jam in time, optimize the traffic situation in the city, and facilitate the residents' travel on the maximum level.

\subsection{Provide Intelligent Transportation Services}

The so-called intelligent transportation, mostly by the use of the positive impact of large data technology, adheres to the basic supporting role. It makes use of big data technology to better play the ability of forecasting on the basis of ensuring the continuous traffic, so as to play an early warning role for the urban road condition in advance. Because the researchers are mostly using the passive guidance at this stage, it shows the disadvantages and lacks the consideration of the driver. Thus, in the follow-up development and continuous improvement, the realization of the intelligent transportation is inevitable, which could select the information provided with the advantage of the Internet. After choosing the most scientific driving route, we gather the wisdom of everyone, and adjust the important role that human and machine can play in the traffic management, to provide the most scientific guidance for people's free travel.

\subsection{Reasonable Adjustment of Traffic Order}

Only a relatively harmonious traffic environment can ensure smooth and unobstructed driving routes, which is meaningful to implement orderly route planning. We appropriately allocate the corresponding resources, adjust the traffic environment, according to the personalized needs proposed by the relevant users, and use scientific intelligent means to design the optimal route of transportation and travel. In addition, in order to achieve effective freight, we must pay attention to the reasonable planning of the related vehicle transportation route. Only in this way can we reach the ideal distribution target in the process of delivery of goods. The integration of wireless sensor is a trend, which can collect the information of vehicle fuel consumption and route more scientifically in time. Through the reasonable monitoring of traffic flow, we analyze the data information which is collected, and the actual congestion of the line is conjectured so as to change the distribution route in a more timely manner. The dispatch center must comprehensively analyze the logistics distribution situation, which is one of the most effective results after the rational use of large data technology. Through the analysis of vehicle information, loading and unloading conditions and weather conditions, the optimal model of vehicle dispatch is designed. We always adhere to the basic principles first in, last-out, and choose the most reasonable and scientific loading and unloading plan.

\section{Conclusion}

Intelligent transportation has been one necessary trend, belonging to one of the key contents in intelligent urban construction. To guarantee the intelligent transportation service to better serve people needs to combine with the advantage of big data technology and ensure the timely handling with those problems of transportation efficiency and traffic jam. Big data technology belongs to the key that leads the rapid development of information era and provides various service for different demands of users, which decides the success or failure of intelligent city to some degree. As for big data technology, it has wide application prospect in intelligent transportation and has positive influence on the development of the whole country on the basis of further optimizing traffic system. Such influence cannot be compared by other technologies.

\section{References}

[1]. The Big Data can "Accurately" Dredge the Congestion and Guarantee the Smoothness — the Cloud Construction Practice of Intelligent Transportation[J]. Information of China construction ,2018 (03) :42-45.

[2]. Bo Zhao. The Development of Intelligent Transportation Based on "Internet Plus" and Big Data Era [J]. Digital Communication World,2017(08):282. 
[3]. Guanglie Mao. Further Improve the Level of Solving Problems Make Efforts to Create the Improvement and Upgrade of Intelligent Transportation[J]. Informational Construction,2017 (05):6-12.

[4]. Jiang Wang. Deputy to the People's Congress Renbin Liao: Push Intelligent Transportation with Internet Plus Big Data[N]. Communication and Information,2017-03-08(A02).

[5]. Jinglin Ma. Challenges and Solution Research Facing Big Data under the Background of Intelligent Transportation[J]. Inner Mongolia science and technology and economy, 2017(02):69-70+119.

[6]. Yamei Zhang, Mingfei Wang and Xiao Ma. The strategic study of big data in the intelligent urban construction of Xinxiang city[J]. Journal of Henan Electromechanical College, 2016, 24(06):912.

[7]. Jian Shao. Intelligent Transportation Service System Based on Mobile Information and Big Data Mining Analysis[J]. Jiangsu Communication,2016,32(05):45-48.

[8]. Yachao Jia, Xianfang Tang and Ru Zhang. Innovative Research on the Application of Intelligent Transportation Big Data under the Background of "Internet Plus" [J]. Modern Economic Information, 2016(05):320.

[9]. Jingyan Ma. Research on Strategies for Solving Traffic Congestion in Intelligent Cities under the Background of Big Data[J]. Computer Knowledge and Technology,2014,10(18):4262-4264.

[10]. Jingyu Ruan. Discussion on the Significant Meaning of Big Data Technology on Promoting Intelligent Transportation in Luohe[J]. Science and Technology Innovation Herald, 2017, 14(22):55-56. 